\title{
GLUTATHIONE: A POSSIBLE LINK TO AUTOPHAGY IN SYSTEMIC LUPUS ERYTHEMATOSUS
}

\author{
${ }^{1}$ Dilip Shah and ${ }^{2}$ Swapan K. Nath \\ ${ }^{1}$ Center for Translational Medicine, Thomas Jefferson University, Philadelphia, PA 19107, USA \\ ${ }^{2}$ Arthritis and Clinical Immunology Research Program, Oklahoma Medical Research Foundation, Oklahoma City, OK 73104, USA
}

Increased free radical formation and altered redox state are of fundamental importance in the pathogenesis of autoimmune diseases including Systemic Lupus Erythematos (SLE). Free radicals are mainly derived from oxygen (Reactive Oxygen Species/ROS) and nitrogen (Reactive Nitrogen Species/RNS) at mitochondria, cellular membranes and the endoplasmic reticulum membrane as physiological responses to a variety of internal and external stress (Shah et al., 2011b). At low levels, they act as signalling molecules and at high levels, they damage organelles, particularly the mitochondria and causes irreversible damage to lipids, DNA and proteins, thus provoking cell death through several modes, including apoptosis and necrosis (Shah et al., 2013). In the last 2 decades, there has been substantial progress in understanding the mechanism of oxidative stress in pathogenesisof SLE and level of intracellular glutathione has been regarded as a checkpoint of oxidative stress (Shah et al., 2014). Altered signal transduction; mTOR is activated by relative depletion of glutathione and supplementation of N-Acetyl Cysteine (NAC), a precursor of glutathione, replenishs intracellular glutathione and inhibitsmTOR signaling and diminished oxidative stress mediated damage in SLE (Fernandez and Perl, 2010). Management of disease through supplement of NAC andrapamycin has been showing promising therapy in SLE patients. Recently, several lines of evidence from genetic, cell biology and lupus animal model studies suggests a pivotal role of autophagy in mediating the occurrence and development of SLE (Alessandri et al., 2012). Interestingly, autophagy is regulated by mTOR pathway and MTOR is activated by relative depletion of glutathione. Thus, redox signaling may provide a link between altered autophagy and depletion of glutathione and autophagy regulation by replenishment of intracellular glutathione may have a therapeutic intervention for the management of disease.
We have shown that changes in the intracellular redox environment of cells, through oxygen-derived free radical production known as oxidative stress, have been reported to be critical for cellular immune dysfunction (Shah et al., 2010), activation of apoptotic enzymes and apoptosis (Shah et al., 2013). Decreased intracellular glutathione levels in the various blood components, including total lymphocytes and its subsets (CD4, CD8 T cell) are strongly associated with disease severity and linked to increase Th1/Th2 cytokine imbalance and lymphocyte apoptosis in SLE patients (Shah et al., 2011a). Similarly, Tewthanom et al. (2009) reported that administration of NAC may be beneficial in mild SLE patients in terms of decreasing lipid peroxidation.

Lai et al. (2012) demonstrates that GSH regulates the elevation of mitochondrial transmembrane potential $\left(\Delta \psi_{\mathrm{m}}\right)$ or Mitochondrial Hyperpolarization (MHP), which in turn activates the mammalian Target Of Rapamycin (mTOR) in lupus T cells. mTOR skews cell death signal processing, modulates T-cell differentiation and, in particular, inhibits the development of $\mathrm{CD}^{+} / \mathrm{CD} 25^{+} / \mathrm{Foxp}^{+}$regulatory $\mathrm{T}$ cells which are deficient in patients with active SLE. Nevertheless, studies of Fernandez and Perl (2010; Lai et al., 2012) are certainly important as they suggest the blockade of mTOR with rapamycin and NACimproves lupus disease activity.

Over recent years, perturbation in autophagy has been implicated in a number of diseases, including systemic lupus erythematosus (Alessandri et al., 2012). Towns et al. (2005) found that serum factors, likely autoantibodies, purified from patients with SLE were able to induce autophagy in neuroblastoma cell lines, providing a further link between autophagy and SLE. Accordingly, several other groups have reported the activated autophagy pathway in $\mathrm{T}$ and $\mathrm{B}$ lymphocytes as a mechanism for survival of autoreactive $\mathrm{T}$ and $\mathrm{B}$ lymphocytes. The

Corresponding Author: Dilip Shah, Center for Translational Medicine, Thomas Jefferson University, Philadelphia, PA 19107, USA 
inhibition of autophagy by blocking mTORsgnalling has been suggested as a novel treatment target in this disease.

Importantly, Lai et al. (2012) has shown that blockade of mTOR with supplementation of NAC reversing glutathione depletion and improving disease activity and fatigue in patients with SLE. NAC treatment promotes expansion of $\mathrm{CD}^{+} \mathrm{CD} 25^{+} \mathrm{FOXP} 3^{+}$T-cell subsets, inhibit anti-DNA antibody production. Indeed, NAC reversed the expansion of $\mathrm{CD}^{-} \mathrm{CD}^{-} \mathrm{T}$ cells, which exhibited the most prominent mTOR activation before treatment with NAC and had been deemed responsible for promoting anti-DNA autoantibody production by B cells. They showed that NACacts as a sensor of $\triangle \Psi \mathrm{m}$, mTOR governs T-cell signalling events implicated in the pathogenesis (Lai et al., 2012). Since, activation of autophagy has been considered to be principally regulated by mTOR pathway and supplementation of NAC block mTOR signalling in SLE patients. It will be intriguing to study the effect of therapeutic supplement of NAC on autophagy in animal models of Lupus and in SLE patients. Such kind of studies encourage to explore more therapeutic potential of NAC which might prove to provide an inexpensive and significant alternative therapy in SLE.

Conflict of Interest: Authors declear no conflict of interest.

\section{REFERENCES}

Alessandri, C., C. Barbati, D. Vacirca, P. Piscopo and A. Confaloni et al., 2012. T lymphocytes from patients with systemic lupus erythematosus are resistant to induction of autophagy. FASEB J., 26: 4722-4732. DOI: $10.1096 / \mathrm{fj} .12-206060$

Fernandez, D. and A. Perl, 2010. mTOR signaling: A central pathway to pathogenesis in systemic lupus erythematosus? Discov Med., 9: 173-178.

Lai, Z.W., R. Hanczko, E. Bonilla, T.N. Caza and B. Clair et al., 2012. N-acetylcysteine reduces disease activity by blocking mammalian target of rapamycin in $\mathrm{T}$ cells from systemic lupus erythematosus patients: A randomized, double-blind, placebocontrolled trial. Arthritis Rheum, 64: 2937-2946. DOI: $10.1002 /$ art.34502
Shah, D., A. Aggarwal, A. Bhatnagar, R. Kiran and A. Wanchu, 2011a. Association between T lymphocyte sub-sets apoptosis and peripheral blood mononuclear cells oxidative stress in systemic lupus erythematosus. Free Radic Res., 45: 559-567. DOI: 10.3109/10715762.2011.555765

Shah, D., A. Wanchu and A. Bhatnagar, 2011b. Interaction between oxidative stress and chemokines: Possible pathogenic role in systemic lupus erythematosus and rheumatoid arthritis. Immunobiology, 216: 1010-1017. DOI: 10.1016/j.imbio.2011.04.001

Shah, D., N. Mahajan, S. Sah, S.K. Nath and B. Paudyal, 2014. Oxidative stress and its biomarkers in systemic lupus erythematosus. J. Biomed. Sci., 21: 23. DOI: $10.1186 / 1423-0127-21-23$

Shah, D., R. Kiran, A. Wanchu and A. Bhatnagar, 2010. Oxidative stress in systemic lupus erythematosus: Relationship to Th1 cytokine and disease activity. Immunol. Lett., 129: 7-12. DOI: 10.1016/j.imlet.2010.01.005

Shah, D., S. Sah, A. Wanchu, M.X. Wu and A. Bhatnagar, 2013. Altered redox state and apoptosis in the pathogenesis of systemic lupus erythematosus. Immunobiology, 218: 620-627. DOI: 10.1016/j.imbio.2012.07.030

Tewthanom, K., S. Janwitayanujit, K. Totemchockcyakarn and D.P.N. Ayudhya, 2009. The effect of high dose of $\mathrm{N}$-acetylcysteine in lupus nephritis: A case report and literature review. J. Clinical Pharmacy Therapeutics, 35: 483-485. DOI: 10.1111/j.1365-2710.2009.01108.x

Towns, R., Y. Kabeya, T. Yoshimori, C. Guo and Y. Shangguan et al., 2005. Sera from patients with type 2 diabetes and neuropathy induce autophagy and colocalization with mitochondria in SY5Y cells. Autophagy, 1: 163-170. DOI: 10.4161/auto.1.3.2068 